\title{
Erratum: Tests of the standard model in neutron beta decay with polarized electrons and unpolarized neutrons and protons [Phys. Rev. D 99, 053004 (2019)]
}

\author{
A. N. Ivanov®, R. Höllwieser, N. I. Troitskaya, M. Wellenzohn, and Ya. A. Berdnikov
}

(Received 1 September 2021; published 17 September 2021)

DOI: 10.1103/PhysRevD.104.059902

In Eq. (18) of this paper in the correlation coefficient $\tilde{\zeta}\left(E_{e}\right) \tilde{K}_{e}\left(E_{e}\right)$ the term $-g_{F}\left(E_{e}\right) E_{e} / m_{e}$ should be replaced by $g_{F}\left(E_{e}\right)$. The same replacement should be done in the definition of the function $h_{n}^{(4)}\left(E_{e}\right)$ in Eq. (A-8). Then, in Eqs. (A-4), (A-6) and (A-7) of this paper in the last term the factor $1 / 4 \beta^{4}$ should be replaced by $1 / 2 \beta^{4}$. Such a replacement allows to simplify the expression in brackets multiplied by the factor $\left(1+\sqrt{1-\beta^{2}}\right)$. Having carried out such a replacement and correct algebraical actions in Eq. (A-8) we obtain the following expressions for the functions $h_{n}^{(3)}\left(E_{e}\right)$ and $h_{n}^{(4)}\left(E_{e}\right)$ :

$$
\begin{aligned}
h_{n}^{(3)}\left(E_{e}\right)= & \lim _{\omega_{\min } \rightarrow 0}\left[g_{\beta_{c}^{-} \gamma}^{(5)}\left(E_{e}, \omega_{\min }\right)-g_{\beta_{c}^{-} \gamma}^{(1)}\left(E_{e}, \omega_{\min }\right)\right]+g_{F}\left(E_{e}\right) \frac{m_{e}}{E_{e}}-g_{F}\left(E_{e}\right) \frac{E_{e}}{m_{e}} \\
= & -\frac{1}{3} \frac{E_{0}-E_{e}}{E_{e}}\left\{\left(1+\frac{1+\beta^{2}}{8 \beta^{2}} \frac{E_{0}-E_{e}}{E_{e}}\right)\left[\frac{1}{\beta} \ln \left(\frac{1+\beta}{1-\beta}\right)-2\right]+\frac{1}{4} \frac{E_{0}-E_{e}}{E_{e}}\right\}-\frac{\beta}{2} \ell n\left(\frac{1+\beta}{1-\beta}\right), \\
h_{n}^{(4)}\left(E_{e}\right)= & \lim _{\omega_{\min } \rightarrow 0}\left[g_{\beta_{c}^{-} \gamma}^{(6)}\left(E_{e}, \omega_{\min }\right)-g_{\beta_{c}^{-} \gamma}^{(1)}\left(E_{e}, \omega_{\min }\right)\right]+g_{F}\left(E_{e}\right) \frac{m_{e}}{E_{e}}+g_{F}\left(E_{e}\right) \\
= & -\frac{1}{3} \frac{E_{0}-E_{e}}{E_{e}}\left\{\left(1+\frac{1+\beta^{2}}{8 \beta^{2}} \frac{E_{0}-E_{e}}{E_{e}}\right)\left[\frac{1}{\beta} \ln \left(\frac{1+\beta}{1-\beta}\right)-2\right]+\frac{1}{4} \frac{E_{0}-E_{e}}{E_{e}}\right\}+\left(1+\sqrt{1-\beta^{2}}\right) \\
& \times\left\{\frac{1}{3} \frac{E_{0}-E_{e}}{\beta^{2} E_{e}}\left[\frac{1}{\beta} \ell n\left(\frac{1+\beta}{1-\beta}\right)-2\right]+\frac{1}{24} \frac{\left(E_{0}-E_{e}\right)^{2}}{\beta^{2} E_{e}^{2}}\left(\frac{3-\beta^{2}}{\beta^{2}}\left[\frac{1}{\beta} \ln \left(\frac{1+\beta}{1-\beta}\right)-2\right]-2\right)+\frac{\sqrt{1-\beta^{2}}}{2 \beta} \ln \left(\frac{1+\beta}{1-\beta}\right)\right\} .
\end{aligned}
$$

For the details of the calculation we refer to the paper (see also Appendix B to Ref. [1]). The corrected functions $h_{n}^{(3)}\left(E_{e}\right)$ and $h_{n}^{(4)}\left(E_{e}\right)$ coincide with the functions $h_{n}^{(1)}\left(E_{e}\right)$ and $h_{n}^{(2)}\left(E_{e}\right)$, defining the radiative corrections of order $O(\alpha / \pi)$ to the correlation coefficients $N\left(E_{e}\right)$ and $Q_{e}\left(E_{e}\right)$, respectively [2].

\section{ACKNOWLEDGMENTS}

This work was supported by the Austrian "Fonds zur Förderung der Wissenschaftlichen Forschung" (FWF) under the Contract No. P31702-N27.

[1] A. N. Ivanov, R. Höllwieser, N. I. Troitskaya, M. Wellenzohn, and Ya. A. Berdnikov, On the structure of the correlation coefficients $S\left(E_{e}\right)$ and $U\left(E_{e}\right)$ of the neutron beta decay, Phys. Rev. C 104, 025503 (2021).

[2] A. N. Ivanov, R. Höllwieser, N. I. Troitskaya, M. Wellenzohn, and Ya. A. Berdnikov, Precision analysis of electron energy spectrum and angular distribution of neutron $\beta^{-}$-Decay with polarized neutron and electron, Phys. Rev. C 95, 055502(E) (2017)

Published by the American Physical Society under the terms of the Creative Commons Attribution 4.0 International license. Further distribution of this work must maintain attribution to the author(s) and the published articles title, journal citation, and DOI. 